\title{
PENSAMIENTO CRÍTICO COMO CRITERIO DE EVALUACIÓN DE ROBUSTEZ ARGUMENTATIVA
}

Cristián Noemi

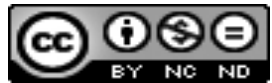

Esta obra está bajo una licencia Creative Commons

Reconocimiento-No Comercial-Sin Obra Derivada 



\title{
PENSAMIENTO CRÍTICO COMO CRITERIO DE EVALUACIÓN DE ROBUSTEZ ARGUMENTATIVA ${ }^{1}$
}

\author{
CRITICAL THINKING AS CRITERION OF EVALUATION OF \\ ARGUMENTATIVE ROBUSTNESS
}

\section{Cristián Noemi}

\begin{abstract}
RESUMEN
El trabajo ha pretendido establecer aspectos de la relación entre tareas de inferencia asociadas a pensamiento crítico y robustez argumentativa del discurso de estudiantes universitarios. En general, dicha relación ha sido investigada muy superficialmente, considerando sólo mínimas variables intervinientes y focalizando la atención preferentemente sobre la estructura sintáctica. Los datos se obtuvieron de dos fuentes a) de la aplicación del test Critical Thinking Test (CTC) y b) de una tarea de escritura de un ensayo argumentativo. Los resultados han permitido identificar el tipo de relación entre las variables.

Palabras clave: Inferencia, robustez argumentativa, discurso, texto, retórica.
\end{abstract}

\begin{abstract}
This study is intended to establish aspects of the relationship between inference tasks associated with critical thinking and argumentative strength in university students' discourse. Traditionally, this relationship has not been studied in depth. Previous studies have only considered minimal variables, and have focused mainly on syntactic structure. Data for our study was obtained from two sources: a) results from the Critical Thinking Test (CTC), and b) a writing assignment, namely a narrative/argumentative essay. Our findings have enabled us to identify the type of association between these variables.
\end{abstract}

Key words: Inference, argumentative strength, discourse, text, rhetoric.

\section{Propósito}

El trabajo tiene como objetivo principal aproximarse a la noción de robustez argumentativa, desde el marco teórico de la lingüística del texto. Para este efecto, se ha explorado tanto en la capacidad que subyace a la actividad de hablar (la 'dínamis') como en el producto que es creado a partir de esta competencia (el 'érgon').

Dr. Cristián Juan Noemi Padilla. Profesor titular, Universidad de La Serena. Chile.

Correo electrónico: cnoemi@userena.cl

Recepción: 27- 07- 2016

Aceptación: 17- 04- 2017 
Desde la primera perspectiva, se sugieren operaciones cognitivas diversas que este saber comprometería durante el ejercicio concreto de la producción discursiva, las que - según se propone- se proyectan en la forma de mayor o menor robustez, textura o densidad argumentativa, sobre el texto.

Desde la segunda, se propone un modelo normativo inicial de robustez del producto basado en la textura relacional de las proposiciones del discurso, y en la noción de coherencia argumentativa que deviene del entramado del mismo.

\section{Metodología}

A fin de explorar respecto de la naturaleza de dominio (Fodor, 1986), tanto de la capacidad 'dínamis' como del producto 'érgon', el trabajo procura especificar aspectos de la eventual relación existente entre las tareas de inferencia -expresadas en términos de pensamiento crítico - y el nivel o grado de robustez argumentativa en estudiantes universitarios.

El trabajo ha seguido un enfoque de naturaleza mixta cuantitativo y cualitativo. A fin de esclarecer las tareas de inferencias de los sujetos evaluados, se aplicó a 80 estudiantes universitarios la prueba Tasks in Critical Thinking (TCT), creada en 1986 por una comisión experta del Educational Testing Service, de Estados Unidos. El instrumento considera tanto la multidimensionalidad del pensamiento como las destrezas cognitivas a priori que éste requiere en una prueba con 15 reactivos de tipo analíticos. Las dimensiones de tipos de inferencias que la prueba considera son tres: indagación, comunicación y análisis.

Con el propósito de determinar el grado de robustez argumentativa de los informantes se ha seguido un procedimiento cualitativo, aplicándose los procedimientos de la grounded theory (Glaser \& Strauss, 1967), a través del software Atlas/ti, versión 5.2. De acuerdo con el modelo, los textos se sintetizaron en categorías conceptuales que aparecieron contextualmente y a las cuales se les asignó un código abierto (Strauss \& Corbin, 1998; Rodríguez, Gil \& García, 1996). Posteriormente, se procedió a agrupar los códigos según sus propiedades; de esta forma se pudo obtener un conjunto de códigos que conformó una unidad superior mediante el recurso de una codificación axial, obteniéndose como resultado un modelo compuesto por relaciones generales entre los elementos, lo que permitió generar una explicación teórica desde la base.

\section{Marco teórico}

\subsection{Pensamiento y lenguaje}

La concepción de una frontera más bien discreta entre las nociones de pensamiento y lenguaje se remonta desde Aristóteles: "Y, así como los signos no son los mismos para todos, tampoco los sonidos son los mismos. Ahora bien, aquello de lo que esas cosas son signos primordialmente, las afecciones del alma, las mismas para todos, y aquello de lo que éstas son semejanzas, las cosas, también las mismas." (Aristóteles, 2001, pp. 25-26), hasta Wittgenstein: "En la proposición, el pensamiento se expresa perceptiblemente por los sentidos." (Wittgenstein, 2003, p. 31); "El signo proposicional aplicado, pensado, es el pensamiento." (Wittgenstein, 2003, p. 46).

La investigación en psicología cognitiva contemporánea, de otra parte, ha puesto de manifiesto que el lenguaje no es la única capacidad cognitiva del ser humano, sino que más bien constituye un módulo de un complejo en el que comparten función al menos la 
percepción, la memoria, la inteligencia y el pensamiento; es decir numerosos subsistemas especializados y relativamente autónomos que, no obstante, interactúan en alguna medida entre sí. En este sentido, se ha propuesto que el lenguaje es un módulo cognitivo (Fodor, 1986) de suerte que su modo de funcionamiento no se encontraría afectado manifiestamente por los demás componentes de la cognición. En este marco, y a partir de evidencias tales como la proteína FoxP2 del cromosoma 7 (Marcus y Fisher, 2003), se postula que el lenguaje sería, por tanto, una habilidad específica, no dependiente del resto de actividades cognitivas.

\subsubsection{Razonamiento natural y lenguaje}

La psicología del desarrollo ha referido que durante los primeros años de vida (etapa de realismo absoluto), los seres humanos asumimos que la representación mental de la realidad se corresponde exactamente con la realidad misma. Con el desarrollo, tanto de las funciones cognitivas como de los rasgos de personalidad, aparece más tarde, entre las funciones cognitivas, la de metacognición y, con ella, cierta capacidad reflexiva que permite generar y cuestionar argumentos.

Según sabemos, el pensamiento reflexivo, al ser metacognitivo, sólo puede darse cuando el contenido mental está registrado simbólicamente, lo que comienza a ocurrir cuando el desarrollo del lenguaje permite la representación y la construcción de conceptos. Cuando se ponen en relación estos conceptos entre sí para obtener una determinada conclusión construimos una suerte de argumentos que pueden o no estar formalizados verbalmente y que son expresión de actividad mental.

En otras palabras, siguiendo a Mercier \& Sperber (2009) admito que los argumentos usados en el razonamiento son el output del mecanismo mental de inferencia. La función de razonar se concibe de este modo como una actividad mental argumentativa puesto que supone una actividad de inferencia conceptual que conlleva no sólo a una nueva representación mental (o conclusión), sino también a representaciones colaterales (o premisas) que proporcionan avales para aceptar la representación mayor.

De este modo se puede concebir el razonamiento tanto como la actividad mental que permite generar un argumento convincente, como la de evaluar y aceptar la conclusión producida por un individuo distinto.

\subsubsection{Razonamiento y pensamiento crítico}

La noción de pensamiento crítico ha sido abordada básicamente desde tres perspectivas suficientemente diferenciadas. En primer lugar, a partir de un enfoque filosófico (Siegel, 2010) que focaliza su atención sobre la 'calidad' del pensamiento desde un punto de vista normativo, en términos de estándares o reglas, i.e., exactitud, claridad, imparcialidad, precisión, lógica, amplitud, relevancia, etc., que ha de alcanzar el sujeto considerado pensador crítico. En segundo lugar, desde los postulados de la psicología cognitiva (Halpern, 2003), centrando más bien la atención en el sujeto real, a través de la investigación del tipo de procesamiento de información que realiza el pensador crítico con el propósito de describir sus fases, tales como análisis, interpretación, definición del problema, formulación de hipótesis, etc. En tercer lugar, desde una óptica pedagógica (Bloom, 1971), a partir de la cual se clasifican las habilidades cognitivas de manera jerárquica en taxonomías graduales.

Las diferentes propuestas, no obstante, coinciden en considerar, de una parte, que el pensamiento crítico es una actividad cognitiva de tipo reflexivo, lo que implica el uso de 
habilidades; luego, que requiere de una determinada disposición (tal como inquisitividad, tendencia a razonar, flexibilidad, y equilibrio); y, finalmente, que requiere de, a lo menos, cierto conocimiento del contexto (información general básica y conocimientos específicos al dominio).

Para los propósitos de este trabajo, siguiendo a Siegel (2010) propongo vincular razonamiento con pensamiento crítico. De acuerdo con su parecer, en efecto, el pensamiento crítico es una manifestación del razonamiento; en tanto que un pensador es crítico un sujeto que se mueve apropiadamente por razones, es decir un individuo que puede evaluar adecuadamente proposiciones y realizar juicios sobre la base de determinadas afirmaciones.

En el marco de este trabajo admito, entonces, que el pensamiento crítico se vincula con la actividad de razonamiento al posibilitar evaluar aquel conocimiento disponible que se registra simbólicamente en el código lingüístico a través de procesos discursivos, preferentemente argumentativos.

\subsection{Robustez argumentativa}

Como tal, la noción de robustez del discurso ha sido abordada muy superficialmente, desde diferentes enfoques teóricos, y centrando la atención preferentemente sobre la microestructura del texto, particularmente sobre su organización sintáctica.

En este tenor, por ejemplo Véliz $(1998,1999)$ relaciona madurez sintáctica con la habilidad para producir unidades lingüísticas del nivel oracional estructuralmente complejas, lo que en su opinión se expresaría en el número de combinaciones y transformaciones que el hablante realiza en el proceso de producción de una determinada secuencia oracional.

Desde otro enfoque epistémico, van Eemeren, Grootendorst y Snoeck (2006), al tratar las distinciones entre una argumentación basada en un argumento único y una argumentación compleja, múltiple, coordinada o subordinada, proponen un modelo de argumentación que considera la complejidad argumentativa en términos del número de argumentos de un texto y de las relaciones que se establezcan entre éstos.

Merlini (2011), por su parte, en un contexto semiótico de mayor envergadura, a partir de las normas de textualidad de Beugrande (1997) relaciona la noción de complejidad textual en cuanto indicio de 'marcación', proponiendo que cualquier secuencia del texto que fuese menos natural o más marcada sería expresión de complejidad, la que en su opinión podría tener como fuente conflicto la cohesión, la coherencia, la intencionalidad, la aceptabilidad, etc.

\subsubsection{Robustez argumentativa en cuanto 'dínamis'}

Con el concepto de robustez argumentativa he pretendido referir, de una parte, en cuanto 'dínamis', aquel conjunto de conocimientos que guardan relación con la capacidad estratégica de que disponen los hablantes para organizar argumentativamente, en distintos grados de textura o densidad, un discurso en lo que concierne al nivel macroestructural del mismo.

Concebida la capacidad discursiva como una competencia, interesa establecer el tipo de conocimiento involucrado en este saber (i.e., razonamiento/pensamiento crítico) que se emplea para desplegar discursivamente la macroestructura de un texto, en distintos niveles de robustez, por medio de un conjunto de proposiciones sucesivamente de nivel inferior.

Admito que las operaciones que suponen la conformación de la robustez argumentativa tienen un sentido precisamente inverso al de las 'macrorreglas' que proponen van Dijk y Kintsch (1983) cuyo propósito, según su parecer, refieren el principio general que ha de seguirse para levantar la macroestructura de un discurso. 
Este tipo de reglas, una suerte de 'macrorreglas de robustez argumentativa', suponen en términos amplios que la información general que comprende la macroestructura se especifica discursivamente en niveles macroestructurales cada vez menores hasta llegar al nivel microestructural donde ya no resulta posible más particularización temática.

Las reglas de producción discursiva que contribuyen a la robustez argumentativa que sugiero y que constituyen, por su parte, una operación en sentido opuesto al de las macrorreglas, son básicamente: 'adjuntar', 'particularizar' y 'especificar'.

La regla 'adjuntar' representa la operación contraria a la macrorregla 'omitir'. A través de la aplicación de ésta podemos 'arropar' al discurso con una serie de proposiciones que son de baja relevancia en relación con la macroestructura y que, precisamente por ello, debieran situarse en un nivel bajo de profundidad macroestructural.

La regla 'particularizar' representa la operación contraria a la regla 'generalizar'. De este modo, a través de su aplicación, podemos descomponer una macroproposición en una serie de proposiciones menores que la impliquen. En cuanto regla de textualización, aparentemente resulta ser de mayor capacidad de densidad, textura o robustez que la regla 'adjuntar'.

La regla 'especificar', finalmente, la concibo como la regla inversa de la operación de 'integrar'. Mediante la puesta en ejecución de esta regla podemos generar discurso en términos de proposiciones que estén subsumidas bajo el 'marco' cultural o guión correspondiente en que se inscribe la macroestructura.

\subsubsection{Robustez en tanto 'érgon'}

Concibo que la competencia lingüística se encuentra conformada por la suma de una serie de saberes independientes (Coseriu, 1992) que han de 'interconectarse' necesariamente entre sí en el momento en que aquella se actualiza en la forma de un discurso concreto. Constreñida por la fuerza de la tradición discursiva, la competencia se proyecta desigualmente en la forma de discurso, afectando por ello la densidad, textura o robustez macroestructural del mismo (van Dijk, 1985), y consecuentemente, su grado de coherencia.

Empleo aquí el término coherencia para hacer mención de la inter-relacionalidad global (macroestructural) en el texto (Halliday y Hasan, 1976; Shapiro y Hudson, 1991) que es dependiente de la estructura formal y de las relaciones que establecen las partes del mismo. Cabe recordar que estas relaciones no son lineales y se establecen en distintos niveles de 'profundidad' del discurso (van Dijk y Kintsch, 1983).

El discurso constituye, en este sentido, un juego de relaciones en el cual las partes actúan como marcadores o indicadores que pautan la densidad, textura o robustez de la macroestructura: "El 'cosmos' del texto está constituido por esta "textura" o juego de enlaces semántico-pragmáticos, dispuestos en diversas capas que se integran unas en otras" (Calsamiglia y Tusón, 2002, p. 219).

En dependencia del grado o calidad de variables de pensamiento crítico que posea o active un determinado hablante se actualizará, en conformidad, un tipo de discurso con mayor o menor grado de coherencia global determinada por la estructura textual del mismo y las relaciones de sus partes, potencialmente medible en términos de robustez o intensidad de la textura del entramado discursivo.

En este sentido, propongo como 'marcadores' de la robustez argumentativa (Ver Tabla1):

1.- El número de proposiciones (p) por secuencia.

2.- El número de p2 por $\mathrm{p}$ ('support'). 
3.- El número de 'explain' por secuencia.

4.- El número de ‘justify’ por secuencia.

5.- El nivel de 'profundidad' macroestructural.

6.- El número de 'topoi' vinculantes.

7.- La naturaleza epistémica del 'topos'.

Tabla 1. Representación de marcadores de robustez argumentativa

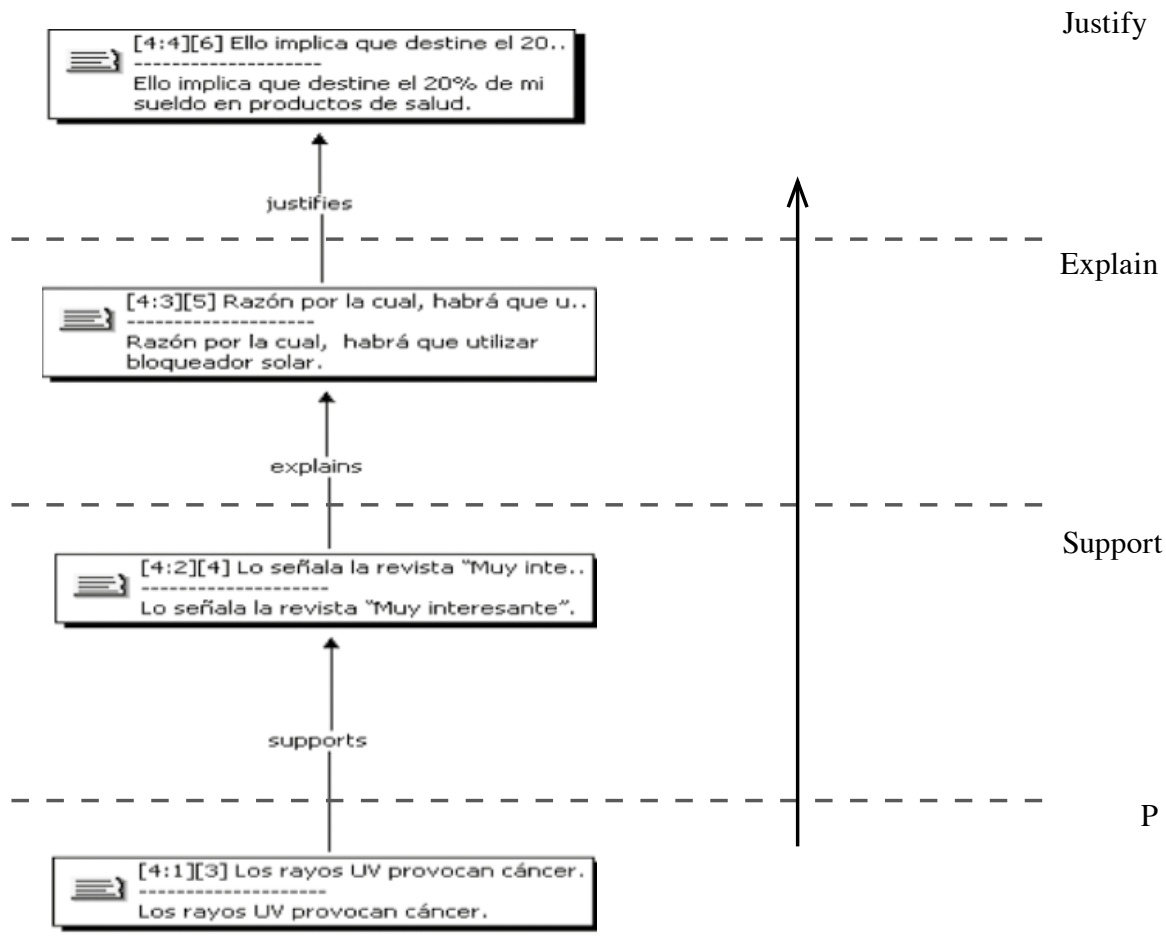

(P), proposición, representa el contenido semántico de una oración y, por tanto, constituye una unidad del nivel inferior de la macroestructura, asociada con la regla de textualización adjuntar y con la dimensión comunicación del test de pensamiento crítico.

Support representa un apoyo argumental de la (P); supone por parte del hablante mayor compromiso cognitivo y está probablemente asociado a mayor nivel de pensamiento crítico.

Explain constituye una explicación extraída a partir del estado de mundo discursivo expuesto por la (P) y avalada por support; supone igualmente actividad cognitiva de mayor envergadura que, junto con support, parecen asociadas con la dimensión cognitiva de indagación medida por el test de pensamiento crítico.

Justify, finalmente, representa una justificación extraída a partir del mundo discursivo generado por (P), support y explain. Supone el mayor esfuerzo cognitivo y se asocia con la dimensión de indagación que mide el test de pensamiento crítico.

\subsubsection{Topos}

A partir de Aristóteles (1990), y en el corpus doctrinario de la práctica totalidad de la teoría retórica clásica, se encuentra una categoría que fue abordada originalmente bajo el concepto de tópica. En opinión del estagirita, a través de los silogismos dialécticos y retóricos 
de 'dicen' los topoi, lo que en opinión de Billig (1988) agregan una cualidad moral a nuestros actos de habla, pues refieren acuerdos de sentido socialmente sancionados.

Aristóteles (1990) distingue entre una tópica mayor y una tópica menor. La tópica mayor atraviesa cualquier discurso en forma transversal, independientemente de su asunto. Así, por ejemplo, algunos topoi como el más y el menos, lo posible y lo imposible, lo que ocurrió o no ocurrió, son transversales a todo discurso, sin importar su tema. De otra parte, la tópica menor, se ocupa de todos aquellos topos que serían adecuados en una ciencia en particular.

En una línea epistémica similar, en el enfoque de Perelman y Olberchts-Tyteca (1989) se propone a este respecto que cuando se pretende fundamentar discursivamente valores o reforzar la intensidad de la adhesión que suscitan, los hablantes pueden recurrir a premisas de carácter general, a las que, siguiendo la tradición otodoxa, denominan igualmente como topoi.

Ducrot (1986), al estudiar el potencial argumentativo de la lengua, propone diferenciar dos tipos de topoi, los extrínsecos y los intrínsecos. Los primeros son aquellos que, en su opinión, guardan relación con la estructura misma de la lengua, y por tanto se manifiestan en palabras gramaticales (relacionales). Los extrínsecos, de otra parte, más próximos a la concepción clásica del concepto, son aquellos que sirven de garante del encadenamiento entre los enunciados al proporcionar un nexo con arreglo a una cierta ética, en un determinado contexto social. En su opinión, corresponden a principios ideológicos, compartidos por una comunidad lingüística más o menos extensa, y que si bien sirven para la construcción arbitraria de representaciones ideológicas, se presentan como si fueran exteriores al locutor.

Por otra parte, Rigotti (2004) proporciona un enfoque mayormente integrado de la noción de topos en el marco de una teoría parcial del lenguaje. En su opinión, el topos constituye una parte del módulo de estrategia argumentativa y cumple una función de elemento generador de enunciado, en cuanto unidad de la dimensión institucionalizada del contexto.

Mi interés se ha focalizado en describir el rol que cumple la categoría de 'topos' en la dinámica del entramado, textura o robustez del discurso. Desde esta perspectiva, el topos cumple la función de proporcionar apoyo o soporte, una suerte de 'anclaje', en el entramado argumental de cada macroestructura (Cf. Tabla 2).

Tabla 2. Anclaje básico

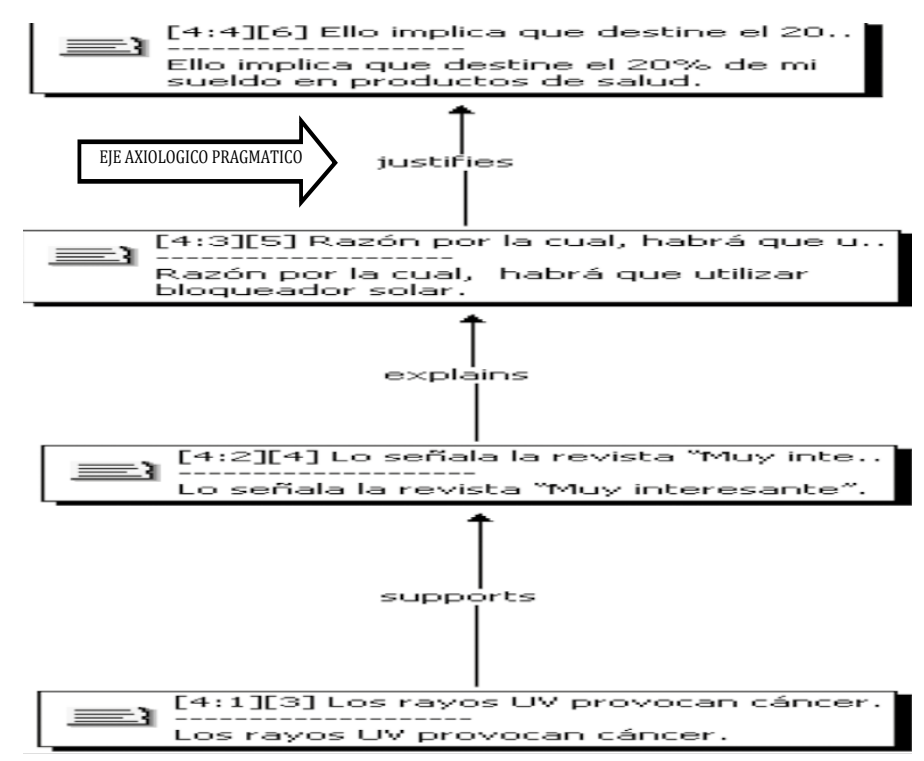


El enfoque pretende concebir a los topoi como un elemento de la competencia de los hablantes naturales que, por tanto, es internalizado y compartido a través de procesos de socialización. En efecto, al aprender una lengua no solo se aprende un repertorio de normas gramaticales, sino que en la adquisición de las reglas del juego de lenguaje se interioriza, también, un determinado sistema de valores que refiere mecanismos de representación de la realidad.

Los topoi están en la base de toda argumentación dado su potencial de objetivar el sistema de valores. Los topoi en cuanto marca, fondo, de la presencia de valores en el discurso cumplen función en cuanto elemento aglutinante que se puede reconocer como marca de coherencia textual, puesto que 'dan la razón', es decir: avalan, respaldan y, refuerzan; esto es, contribuyen a la densidad, textura, y consecuentemente a la coherencia argumentativa y verosimilitud del discurso.

\subsubsection{Ejes axiológicos}

A partir de Aristóteles (2006), y en dependencia del tipo de discurso a través del cual se manifiesten preferentemente, distinguimos tres ejes axiológicos: el eje cardinal, el teologal y el pragmático. La noción de 'eje axiológico’ pretende referir el dominio fundamental de los tipos de valores que se plasman en un determinado discurso.

El eje cardinal debe su nombre a las cuatro virtudes llamadas cardinales o también primarias: la prudencia, la fortaleza, la humildad y la tolerancia (Aristóteles, 2006). El eje teologal toma su denominación de las virtudes llamadas del mismo nombre que incorpora el cristianismo: la fe, la esperanza y la caridad. Por último, el eje pragmático debe su designación al llamado logos pragmático (Aristóteles, 2013), que a diferencia del logos apofántico, más propio de la ciencia y que descansa en la primacía que adquiere la lógica proposicional, reposa más bien en la acción.

\section{Hallazgos}

Con el propósito de mostrar la relación que se ha develado entre capacidad de inferencia y robustez discursiva, se presentan a continuación (cf. Tabla 2 y Tabla 3 ) dos ejemplos del corpus con los puntajes obtenidos en las dimensiones 'pensamiento crítico' y 'robustez argumentativa', respectivamente, que a su vez son prototípicos de la tendencia general que emana del corpus.

El sujeto 1 representado en la Tabla 3 (Anexo 1, III Parte, apartado 3A.) categorizado como 'deficiente' en términos de la dimensión de pensamiento crítico al alcanzar un puntaje total de 17 puntos, 8 en la categoría de análisis, 5 en la de indagación y un comportamiento 'normal' en la de comunicación, a través de las operaciones cognitivas que he denominado 'macrorreglas de robustez argumentativa', genera un discurso de tipo descriptivo explicativo con 11 proposiciones (p1) en la base, apoyadas (rotuladas como 'support' por el software) por 10 (p2) en el siguiente nivel de profundidad macroestructural. Se observa en tanto estrategia discursiva que algunas (p1) están soportadas por más de una (p2), y que el topos nexual vinculante es de tipo causal, por lo que el sujeto se sitúa epistémicamente en un eje de naturaleza pragmático. Con arreglo a la propuesta, el texto manifiesta igualmente baja robustez argumentativa, y consecuentemente baja coherencia, en virtud del débil tejido, entramado o densidad discursiva, representado por la escasa profundidad macroestructural y la ausencia de relaciones sustantivas entre proposiciones. 
Tabla 3. Sujeto 1

a) Dimensión pensamiento crítico, Sujeto 1

\begin{tabular}{|l|l|}
\hline Pensamiento crítico & Deficiente \\
\hline Puntaje total & 17 \\
\hline Categoría comunicación & Normal \\
\hline Categoría análisis & 8 Puntos \\
\hline Categoría indagación & 5 Puntos \\
\hline
\end{tabular}

b) Dimensión robustez argumentativa, Sujeto 1

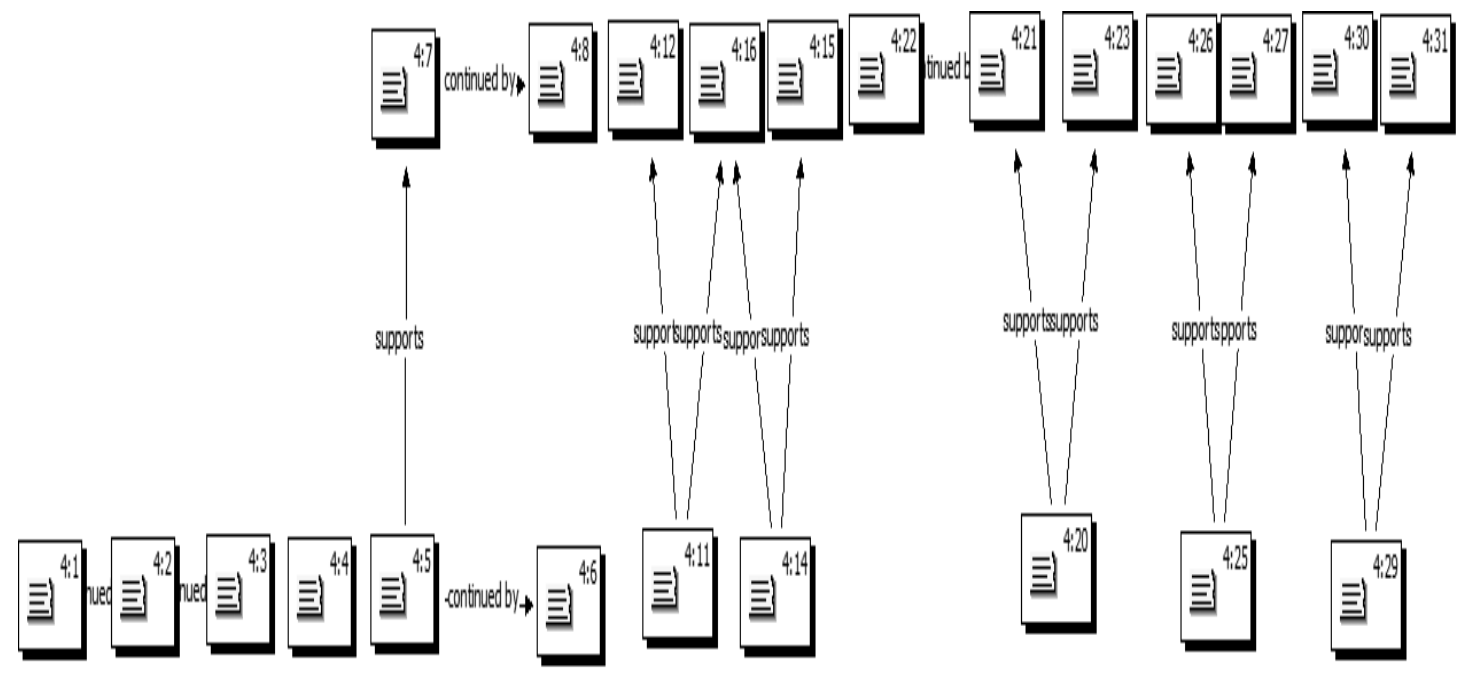

El sujeto 2 representado en la Tabla 3 (Anexo 2, III Parte, apartado 3A.) categorizado como 'alto' en términos de la dimensión de pensamiento crítico por lograr un puntaje total de 31 puntos, 12 en la categoría de análisis, 14 en la de indagación y un desempeño 'alto' en la de comunicación, a través de las 'macrorreglas de robustez argumentativa', genera un discurso de tipo descriptivo explicativo con 3 proposiciones (p1) en la base, soportadas por 4 (p2) en el segundo nivel de complejidad (rotulado por el software como support). Al contrario del sujeto 1, éste compromete mayor densidad discursiva al avanzar con macrorreglas de mayor envergadura cognitiva hacia un segundo nivel de complejidad macroestructural rotulado por el software como 'explain' que garantiza en virtud de la textura mayor coherencia global. En efecto, las 4 (p2) están garantizadas por 7 (p3) que, a su vez, establecen diversas relaciones según se observa en la Tabla 2 (cruces, haces, etc.). El sujeto 2 aplica macrorreglas de aun mayor compromiso cognitivo hasta alcanzar un tercer nivel de robustez argumentativa rotulado como 'justifies' por el software, por lo que, en términos de textura, el discurso alcanza un grado de complejidad y coherencia estimables. El sujeto 2 emplea topos nexuales vinculantes de tipo causal y de ejemplo, por lo que se sitúa epistémicamente también en un eje de naturaleza pragmático. 
Tabla 4. Sujeto 2

a) Dimensión pensamiento crítico, Sujeto 2

\begin{tabular}{|l|l|}
\hline Categoría pensamiento crítico & Alto \\
\hline Puntaje total & 31 \\
\hline Categoría comunicación & Alto \\
\hline Categoría análisis & 12 Puntos \\
\hline Categoría indagación & Puntos \\
\hline
\end{tabular}

b) Dimensión robustez argumentativa, Sujeto 2

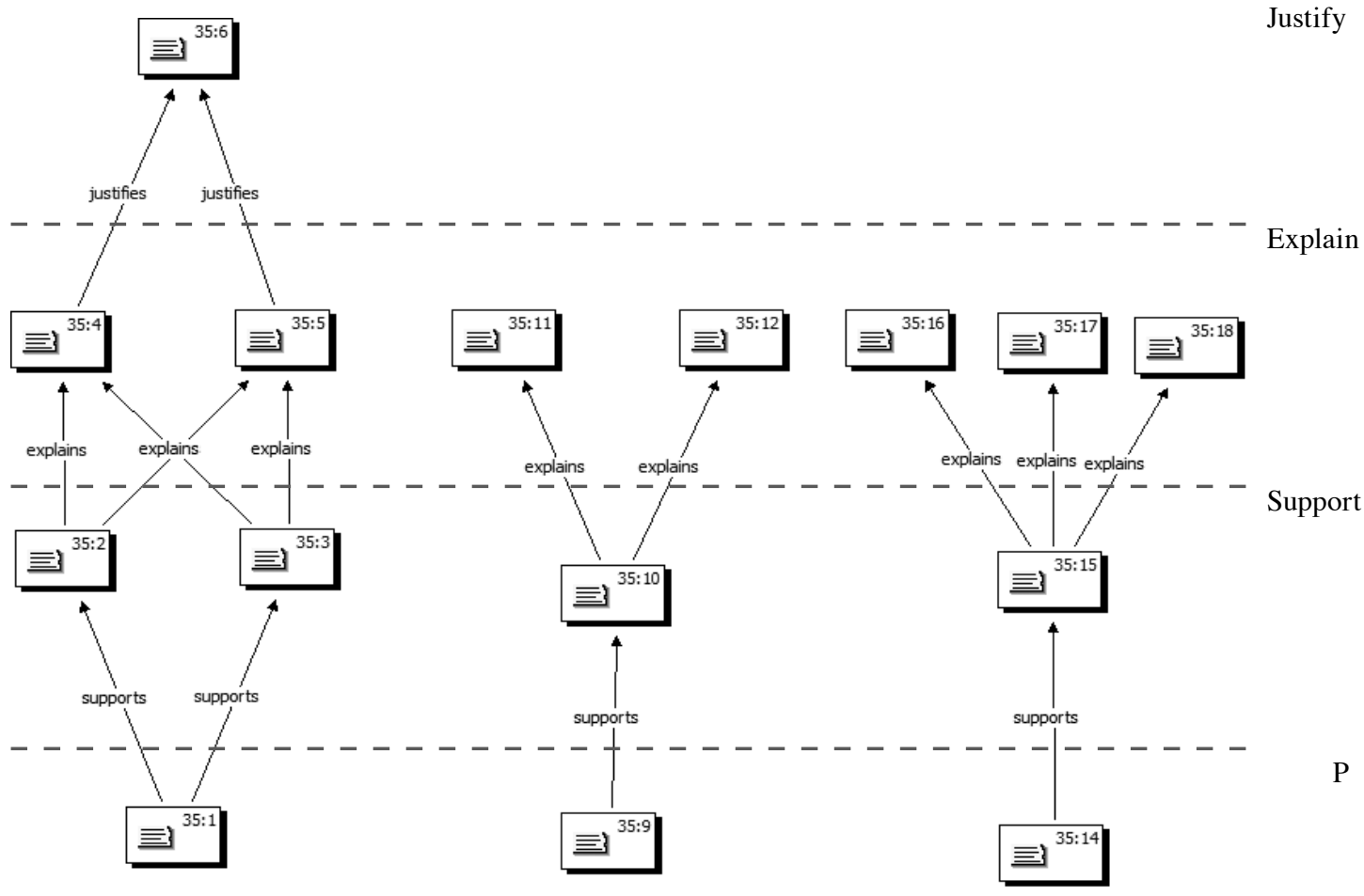

\section{Conclusiones}

El trabajo ha permitido proponer inicialmente ciertas operaciones cognitivas asociadas a la competencia linguiística, 'adjuntar', 'particularizar' y 'especificar', cuya función según se ha sugerido es desplegar textualmente, en cuanto 'dínamis', la macroestructura textual provocando con ello distintos grados de densidad, textura o robustez discursiva.

En un sentido similar, a partir de los datos se pudo sugerir un modelo de 'robustez argumentativa', el cual ha pretendido referir, en cuanto 'érgon', los diversos grados de textura o densidad del discurso, en lo que concierne al nivel macroestructural del mismo.

El cruce entre la información proporcionada por el test Tasks in Critical Thinking y la obtenida por medio del modelo de robustez discursiva ha permitido especificar aspectos de la relación existente entre pensamiento y lenguaje, particularmente en lo que guarda relación con la especificidad de los dominios de estos módulos de la cognición. 
A partir de la muestra sometida a análisis, resulta posible sostener que claramente existe relación de determinación entre la capacidad de inferencia y la robustez argumentativa. De las dimensiones cognitivas medidas en forma cuantitativa, las que mayores determinaciones establecen con robustez argumentativa son las capacidades de análisis y síntesis.

El trabajo permitió aislar una función de los topoi no reconocida en la literatura y que según se ha mostrado cumple la función formal de elemento aglutinador entre niveles constitutivos de la macroestructura, contribuyendo de este modo a la densidad, textura, y robustez discursiva, y consecuentemente, a la coherencia semántica.

Los resultados, finalmente, sugieren que el modelo de robustez pareciera un instrumento estimable al momento de usarse como técnica correspondiente al análisis del discurso.

\section{Notas}

1. Este trabajo se enmarca en el proyecto de investigación DIDULS regular $\mathrm{N}^{\mathrm{o}} 1130584$.

\section{Bibliografía}

Aristóteles. (1990). Retórica. (4 ed.). Tovar, A.(Ed.). Madrid: Centro de Estudios Constitucionales. Aristóteles. (2001). Tratados de lógica. México: Editorial Porrúa, S. A.

Aristóteles. (2006). Ética a Nicómaco. Buenos Aires: Gradifco.

Aristóteles. (2013).Perí hermeneias. (E. M. Edghill, tr.).eBooks@Adelaide The University of Adelaide Library, University of Adelaide.

Beugrande, R. (1997). Introducción a la lingüística del texto. Barcelona: Ariel.

Billig, M. (1988). Common-places of the British Royal Family: a rhetorical analysis of plain and argumentative sense. Text. 8 (3), 191-217.

Bloom, B., et ál. (1971). Taxonomía de los objetivos de la educación: la clasificación de las metas educacionales : manuales I y II. (M. Pérez-Rivas, tr.). Buenos Aires: Centro Regional de Ayuda Técnica: Agencia para el Desarrollo Internacional (A.I.D).

Calsamiglia, H y Tusón, A. (2002). Las cosas del decir: manual de análisis del discurso. Barcelona: Ariel.

Coseriu, E. (1992). Competencia lingüística. Elementos de la teoría del hablar. Madrid: Gredos.

Ducrot, O. (1986). El decir y lo dicho. Polifonía de la enunciación. Barcelona: Ediciones Paidós.

Fodor, J. (1986). La modularidad de la mente. Madrid: Ediciones Morata.

Glaser, B. \& Strauss, A. (1967). The discovery of grounded theory: strategies for qualitative research. New York: Aldine de Gruyter.

Halliday, M. y Hasan, R. (1976). Cohesion in English. London: Longman.

Halpern, D. F. (2003). El pensamiento y el conocimiento: una introducción al pensamiento crítico. (4 ed.). Nueva Jersey: Editores de Lawrence Erlbaum Associates.

Marcus, G. F. y Fisher, S. E. (2003). FOXP2 in focus: what can genes tell us about speech and language? Trends. Cognitive Science. 7, 257-262. 
Mercier, H., \& Sperber, D. (2009). Intuitive and reflective inferences. Por J. S. B. T. Evans \& K. Frankish (Eds.). In Two Minds. (149-170). New York: Oxford University Press.

Merlini, L. (2011). A 'natural' approach to text complexity. Poznań Studies in Contemporary Linguistics. 47 (2), 203-236.

Perelman, Ch. y Olbrechts-Tyteca, L. (1989) Tratado de la argumentación. La nueva retórica. Madrid: Gredos.

Rigotti, E. (2004) Intercultural Communication: A linguistic and argumentative approach. Lugano: Master in Intercultural Communication (MIC) USI.

Rodríguez, G., Gil J. \& García E. (1996). Metodología de la investigación cualitativa. Málaga: Aljibe.

Shapiro, L. R., y Hudson, J. A. (1991). Tell me a make-believe story: Coherence and cohesion in young children's picture-elicited narratives. Developmental Psychology. 27, 960-974.

Siegel, H. (2010). Critical Thinking. Por P. Peterson, E. Baker y B. McGaw (Eds.). International Encyclopedia of Education. (141-145). Oxford: Elsevier.

Strauss, A. \& Corbin, J. (1998). Basics of qualitative research. Techniques and procedures for developing grounded theory. London: Sage.

Véliz, M. (1988). Evaluación de la madurez sintáctica en el discurso escrito. RLA. Revista de Lingüista Teórica y Aplicada. 26, 105-141.

Véliz, M. (1999). Complejidad sintáctica y modo del discurso. Estudios Filológicos. 34, 181-192.

van Eemeren, F. H., Grootendorst, R., y Snoeck Henkemans, F. (2006). Argumentación: análisis, evaluación, presentación. Buenos Aires: Biblos.

van Dijk, T. (1985). Handbook of Discourse Analysis. London: Academic Press.

van Dijk, T. y Kintsch, W. (1983). Strategies of discourse comprehension. New York: Academic Press.

Wittgenstein, L. (2003). Tractatus logico-philosophicus. Madrid, Alianza Editorial.

\section{Anexos}

Anexo sujeto 1

III PARTE

Instrucción Específica: use la mayor cantidad de información que se halle disponible. Para ello utilice los dibujos 1 y 2 así como algunos antecedentes del documento 1

3A. Desarrolle un ensayo sobre la situación vivida en Caleta de Ojiva a raíz de la aparición del Fenómeno del Niño y las medidas adoptadas por las autoridades locales, identificando variables, estableciendo relaciones y reconociendo la coherencia de las acciones.

Las caletas viven principalmente de las actividades pesqueras, ya sea extracción y comercialización de productos del mar, como también restaurantes, ferias artesanales, entra 
otras actividades. En la caleta ojiva ubicada en la III región se vive una situación en la cual los habitantes de la caleta se ven directamente afectados.

El fenómeno del niño es un fenómeno climático que provoca una alza en la temperatura del agua, como lo que provoco en la caleta de ojiva donde la temperatura del agua marina sufrió un alza de $5^{\circ} \mathrm{C}$ lo que neutralizo los efectos de la riqueza fitoplactónica, organismo el cual es la principal base de la cadena alimenticia de la fauna marina.

Como vemos en el dibujo número 2, en una situación de ausencia del fenómeno del niño, los pescadores realizaban su trabajo en completa normalidad, a diferencia del dibujo número 1 donde, al haber presencia del fenómeno niño, la actividad pesquera se vio directamente afectada al disminuir considerablemente la concentración de peces en el mar.

El municipio de tal-tal decidió actuar e implanto dos medida para ayudar a las familias de la caleta, las cuales viven de la extracción de peces, para que sus ingresos no se vieran afectados. Ambas medidas logran que las familias mantengan ingresos para su calidad de vida, y a la vez incentiva el turismo en la zona al potenciar también áreas paralelas a la extracción de peces, pero también hay que dimensionar el alza que provocara la falta de peces en los precios de los productos, lo cual también podrá provocar un efecto negativo, tanto en la situación económica de las familias de la caleta, como en el turismo de la zona.

Anexo sujeto 2

III PARTE

Instrucción Específica: use la mayor cantidad de información que se halle disponible. Para ello utilice los dibujos 1 y 2 así como algunos antecedentes del documento 1

3A. Desarrolle un ensayo sobre la situación vivida en Caleta de Ojiva a raíz de la aparición del Fenómeno del Niño y las medidas adoptadas por las autoridades locales, identificando variables, estableciendo relaciones y reconociendo la coherencia de las acciones.

Se le denomina fenómeno del niño a la alteración de la corriente de Humboldt que consiste en el calentamiento periódico de las aguas marinas, este conlleva una serie de consecuencias no solo económicas (disminución de recursos ícticos), si no también climáticas (inundaciones, precipitaciones, etc.), es por esto que la mayoría de las autoridades han optado establecer planes que permitan afrontar este fenómeno, uno de ellos es la determinación de nuevas zonas de extracción y la capacitación de nuevas tareas a los pescadores, los cuales permiten que las personas que trabajan en ese sector (primario extracción) puedan desarrollar otras labores para subsistir económicamente.

En síntesis podemos mencionar que la llegada de este fenómeno a nuestras aguas es catastrófico no solo porque modifica el ambiente marino, sino que también es perjudicial en nuestra economía, en la biomasa y el clima trayendo una serie de consecuencias que son difíciles de afrontar. 
